\title{
PROOF OF A CONJECTURE OF ROUTLEDGE
}

\author{
SHIH-CHAO LIU
}

According to Routledge [1], every g.r. (general recursive) function of one variable can be expressed as $g(\phi(a))$. Here $\phi(a)$ is a p.r. (primitive recursive) function and $g(a)$ is a function defined by the schema

$$
\begin{aligned}
& g(n)=m, \\
& g(a)=h(a, g(\delta(a))), \quad \text { for } a \neq n
\end{aligned}
$$

where $h(a, b)$ and $\delta(a)$ are p.r. functions and $\delta(a)<a$ for $a \neq n$ in a well-ordering, of order type $\omega$, of the natural numbers with $n$ as the first element. Routledge also conjectured that not every g.r. function can be expressed as $\phi(g(a))[1]$.

The purpose of this note is to give a proof for this conjecture by actually constructing a g.r. function $\psi(a)$ and then showing that for any p.r. function $\phi(a)$ and any function $g(a)$ defined by the preceding schema, $\psi(a)$ is not identically equal to $\phi(g(a))$. An argument for which $\psi(a) \neq \phi(g(a))$ can be actually found by using the method in the proof, provided that the well-ordering of type $\omega$ involved in the definition of $g(a)$ is itself constructively given. In this sense, the proof can be regarded as a constructive one.

Definition. A finite sequence of natural numbers

$$
a_{0}, a_{1}, \cdots, a_{r}
$$

is called a w.o. (well-ordered) segment of rank $p$, if and only if $r>0$, $a_{r}=p, a_{i} \neq a_{j}$ for $i \neq j$, and $a_{i}<a_{r}$ for $i<r$.

Proof of the Conjecture. Let $\varphi_{1}(y, a)$ and $\varphi_{2}(y, a, b)$ be two enumerating functions of p.r. functions of one variable and two variables respectively [2]. Let $\tau(a, b, c, d, e), \tau_{1}(a), \tau_{2}(a), \tau_{3}(a), \tau_{4}(a), \tau_{5}(a)$ be six p.r. functions such that for any five given numbers $a, b, c, d, e$, a unique number $x$ exists so that $\tau_{1}(x)=a, \tau_{2}(x)=b, \tau_{3}(x)=c, \tau_{4}(x)=d$, $\tau_{5}(x)=e$ and $\tau(a, b, c, d, e)=x$. (These functions can be constructed readily by using the p.r. functions $\sigma(a, b), \sigma_{1}(a), \sigma_{2}(a)$ as defined by Peter [3].) Now, let a g.r. function $\psi(a)$ be constructed as follows:

The first step. Put $\psi(0)=0$.

The $(p+1)$ th step $(p>0)$. There are obviously only a finite number

Received by the editors August 21, 1959 and, in revised form, January 18, 1960. 
of w.o. segments of rank $p$. Let this finite number be $\pi(p)$. Consider any one of such segments $a_{0}, a_{1}, \cdots, a_{r}$. We shall determine a number $x$ from this segment and then use this number for the evaluation of $\psi(p)$. Let $x$ be the least number $y$ not being used at the previous steps, such that $y$ satisfies the conditions:

$$
\begin{aligned}
\tau_{1}(y) & =a_{0}, \\
\varphi_{1}\left(\tau_{5}(y), a_{i}\right) & =a_{i-1}, \quad \text { for } i=1, \cdots, r .
\end{aligned}
$$

It is noted that this number $x$ can always be found. For, obviously there exists a number $u^{*}$ such that the p.r. function $\varphi_{1}\left(u^{*}, a\right)$ has the property $\varphi_{1}\left(u^{*}, a_{i}\right)=a_{i-1}$ for $i=1, \cdots, r$; then the expression $\tau\left(a_{0}, l, s, t, u^{*}\right)$ with $l, s, t$ as parameters gives infinitely many numbers all satisfying the conditions (i) and (ii) when one of the parameters, say, $l$ runs over all the natural numbers.

We use the number $x$ to define a partial recursive function $k(a)$ by

$$
\begin{aligned}
k(a) & =\varphi_{1}\left(\tau_{3}(x), g(a)\right), \\
g\left(\tau_{1}(x)\right) & =\tau_{2}(x), \\
g(a) & =\varphi_{2}\left(\tau_{4}(x), a, g\left(\varphi_{1}\left(\tau_{5}(x), a\right)\right)\right), \quad \text { for } a \neq \tau_{1}(x) .
\end{aligned}
$$

Since $p=a_{r}$ and $x$ satisfies the conditions (i) and (ii), then it is seen that the value of $k(a)$ for $a=p$ is uniquely determined by the above equations and can be evaluated in a finite number of steps.

Since there are $\pi(p)$ w.o. segments of rank $p$, then we can determine $\pi(p)$ numbers $x_{p, 1}, \cdots, x_{p, \pi}$ and then use them to define $\pi(p)$ partial recursive functions $k_{p, 1}(a), \cdots, k_{p, \pi}(a)$ respectively in a similar way as described above. To conclude this step, we let

$$
\psi(p)=1+k_{p, 1}(p)+\cdots+k_{p, \pi}(p) .
$$

The function $\psi(a)$ as constructed above is effectively calculable for each argument $a$ and consequently $\psi(a)$ is a g.r. function. (See, for example, [4].)

Let $\phi(a)$ be any p.r. function and $g(a)$ be any function defined at the beginning of this note. A number $w$ is called a favorable argument of the function $\phi(g(a))$, if for any number $i>0, \delta^{i}(w)<w$, if $\delta^{i}(w)<w$. We shall show in the following that there are infinitely many such favorable arguments and among them there is at least one argument for which $\psi(a) \neq \phi(g(a))$.

Given any natural number $q$, we find the number $k$ such that $q$ is the $k$ th element in the ordering $\prec$. Find the greatest number $z$ among the first $k$ elements in the ordering $\prec$ and again find the greatest number $w$ among the $(k+1)$ th, $\cdots,(k+z+2)$ th elements in the 
ordering $\prec$. If $\prec$, the well-ordering of type $\omega$, is constructively given, the numbers $k, z$ and also $w$ can be actually found. It can be easily verified that $q<w, n<w$ and that for any $y, y<w$, if $y<w$. Since $\delta(a)<a$ for $a \neq n$, then for any $i>0, \delta^{i}(w)<w$, if $\delta^{i}(w)<w$. Consequently $w$ is a favorable argument of $\phi(g(a))$. Since $w$ is greater than the arbitrarily given number $q$, then the number of such favorable arguments is infinite.

We find three numbers $s, t, u$, such that $\varphi_{1}(s, a)=\phi(a), \varphi_{2}(t, a, b)$ $=h(a, b)$ and $\varphi_{1}(u, a)=\delta(a)$. Let $x_{0}=\tau(n, m, s, t, u)$. Suppose $w$ be any favorable argument of $\phi(g(a))$. Since $n$ is the first element in the ordering $\prec$, we can find a number $r$ which is the least number $i$ such that $\delta^{i}(w)=n$. Then the sequence $\delta^{r}(w), \cdots, \delta(w), w$ is a w.o. segment of rank $w$ with its initial term $\delta^{r}(w)=n$. Denote this w.o. segment by $a_{0}, \cdots, a_{r}$. Then we have $\tau_{1}\left(x_{0}\right)=n=a_{0}$ and $\delta\left(a_{i}\right)$ $=\varphi_{1}\left(\tau_{5}\left(x_{0}\right), a_{i}\right)=a_{i-1}$ for $i=1, \cdots, r$. Hence $x_{0}$ satisfies the conditions (i) and (ii). According to the recipe for the construction of $\psi(a)$, the arbitrarily given favorable argument $w$ has the property that if $x_{0}$ is used neither at the $(w+1)$ th step nor at any previous step, then there exists a number which is used at the $(w+1)$ th step and is less than $x_{0}$. Among the infinitely many favorable arguments we can find $x_{0}+1$ of them, say, $w_{1}, \cdots, w_{x_{0}+1}$. It can not be that for every $j\left(1 \leqq j \leqq x_{0}+1\right), x_{0}$ is used neither at the $\left(w_{j}+1\right)$ th step nor at any other step preceding to it. For if it were the case, there would be $x_{0}+1$ distinct numbers all less than $x_{0}$. This is impossible. Hence there must be a step, say, the $(p+1)$ th step (where $p \leqq$ some $w_{j}$ ) at which $x_{0}$ is used for the evaluation of $\psi(p)$. This number $p$ is, of course, still a favorable argument of $\phi(g(a))$.

The number $x_{0}$ is, then, one among the $\pi(p)$ numbers $x_{p, 1}, \cdots, x_{p, \pi}$ and is used to define one of the $\pi(p)$ partial recursive functions, say, $k_{p, j}(a)$. By definition, $\psi(p)$ is greater than $k_{p, j}(p)$ at least by 1 . In fact, $k_{p, j}(a)$ is just the same function as $\phi(g(a))$. Thus $\psi(a)$ is not identically equal to $\phi(g(a))$. This completes the proof of the conjecture of Routledge.

\section{REFERENCES}

1. N. A. Routledge, Ordinal recursion, Proc. Cambridge Philos. Soc. vol. 49 (1953) pp. 175-182.

2. R. Peter, Rekursive Funktionen, Budapest, Akademiai Kiado, 1951. $42-60$.

3. - Konstruktion nichtrekursiver Funktionen, Math. Ann. vol. 111 (1935) pp.

4. S. C. Kleene, General recursive functions of natural numbers, Math. Ann. vol. 112 (1936) pp. 727-742.

Academia Sinica, Taiwan, Chrna 